\title{
AC operation of large titanium sublimation pumps in a magnetic field: results of the test stand for the W7-X neutral beam injectors
}

G. Orozco, M. Froeschle, B. Heinemann, C. Hopf, R. Nocentini, R. Riedl, A. Staebler

Max-Planck-Institute for Plasma Physics, Boltzmannstraße 2, 85748 Garching, Germany

E-mail contact of main author: guillermo.orozco@ipp.mpg.de

\begin{abstract}
A neutral beam injection (NBI) system is being built for the Stellarator experiment Wendelstein 7-X (W7-X) currently under construction at IPP Greifswald. The NBI system consists of two injectors which are essentially a replica of the system present in the Tokamak experiment ASDEX-Upgrade at IPP Garching. A vacuum system with high pumping speed and large capacity is required to ensure proper vacuum conditions in the neutral beam line. For this purpose, large titanium sublimation pumps (TSP) are installed inside the NBI boxes, consisting of $4 \mathrm{~m}$ long hanging wires containing Ti and the surrounding condensation walls. The wires are DC ohmically heated up with $142 \mathrm{~A}$ to Ti sublimation temperature. A TSP system has been operated since many years in the AUG - NBI system, sublimating $\mathrm{Ti}$ in the pauses between the plasma discharges, when no magnetic field is present. However, at W7-X the superconducting coils generate a magnetic field permanently during experimental campaigns, whose stray B field with a maximum of $30 \mathrm{mT}$, affects the TSPs. Operated with DC, the wires would be deflected against the surrounding panels due to the Lorentz force. A simple possible solution is heating with $A C$, which reduces the wire deflection amplitude, inducing a risky wire oscillation. The feasibility of the $A C$ operation in an equivalently strong $B$ field such as the stray B field around W7-X has been demonstrated in a test stand for different AC waveforms and frequencies. Several test campaigns have shown no qualitative difference in the pumping properties between AC and DC operation of the TSP and no critical dynamic behaviour of the wires.
\end{abstract}

\section{Keywords}

Wendelstein 7-X; Neutral Beam Injection; Titanium sublimation pump; Alternating current; Magnetic field.

\section{Introduction}

Neutral beam injection (NBI) is one of the systems used in experimental fusion devices to heat the magnetically confined plasma to a temperature of several ten million degrees Celsius. At IPP Garching a positive-ion-based NBI system has been used on the ASDEX-Upgrade (AUG) Tokamak since 1993 and a similar system is under construction for the Stellarator type device at IPP Greifswald, Wendelstein 7-X (W7-X) [1]. The AUG-NBI system consists of two injectors with four positive ion sources each, for $\mathrm{H}^{+}$and $\mathrm{D}^{+}$. The southeast (SE) injector operates with arc discharge ion sources and the northwest (NW) injector with radio frequency driven sources. The ion beams are accelerated with $60 \mathrm{kV}$ (SE) or $93 \mathrm{kV}(\mathrm{NW})$ for $\mathrm{D}^{+}$during a maximum of 10 seconds and are finally neutralized in order to penetrate the strong magnetic field ( $B$ field) of the device. The total heating 
power of the two injectors together is up to $13 \mathrm{MW}$ for $\mathrm{H}^{0}$ and up to $20 \mathrm{MW}$ for $\mathrm{D}^{0}$. During the $10 \mathrm{~s}$ NBI pulses, a gas feed of up to $30 \mathrm{~Pa}^{*} \mathrm{~m}^{3} / \mathrm{s}\left(\mathrm{H}_{2} / \mathrm{D}_{2}\right)$ is required for beam operation, while the gas pressure must remain below $10^{-2} \mathrm{~Pa}$ downstream in any section of the beam line in order to avoid high re-ionization losses and eventually a severe beam blocking event [2]. A fast pumping system $\left(3^{*} 10^{3} \mathrm{~m}^{3} / \mathrm{s}\right.$ for $\mathrm{H}_{2}$ ) with a large capacity is therefore required for each injector. At IPP it has been traditionally achieved with titanium sublimation pumps (TSP). In the TSP of the AUG-NBI, Ti is sublimed from $4 \mathrm{~m}$ long hanging wires, DC ohmically heated with $142 \mathrm{~A}$, from where $\mathrm{Ti}$ atoms sublime and then are deposited on water cooled panels, acting as gas molecule getter. W7- $X$ is a superconducting machine with coils that keep a B field of up to $3 \mathrm{~T}$ permanently inside the machine, uninterrupted during the experimental day. The stray $B$ field in the torus hall will reach values up to around $30 \mathrm{mT}$ at the front region of the NBI system (fig. 1) [3]. In presence of such strong permanent $B$ field, the DC operated hanging wires would be unacceptably deflected by the Lorentz force and contact other wires or surrounding structures. A possible simple solution is to heat by AC instead of

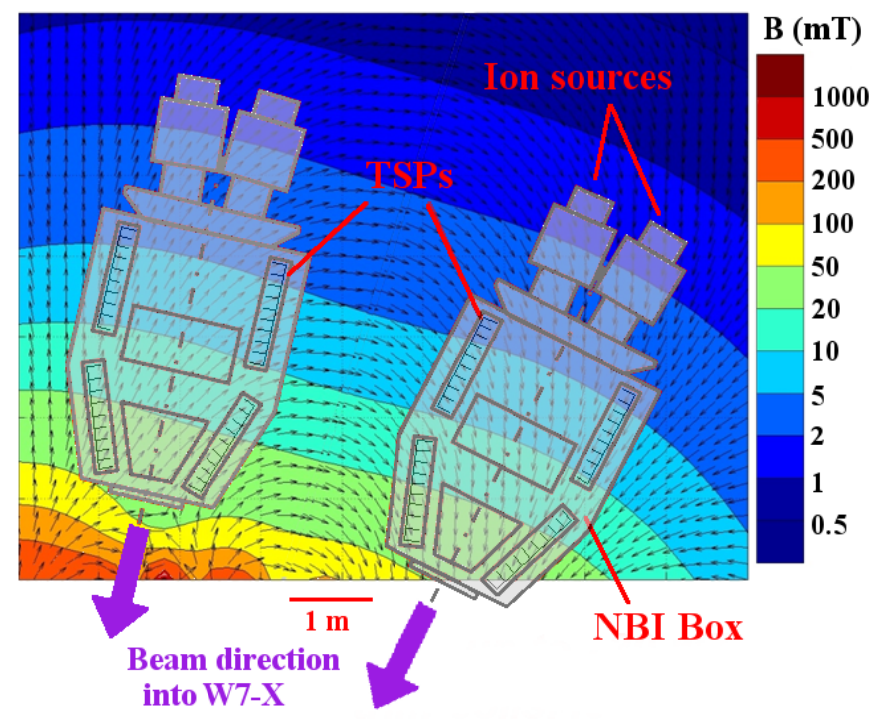

Figure 1: Top view of the two NBI injectors with four ion sources and four TSPs each. The contour plot shows the simulation of the stray $B$ field of W7-X in the surroundings of the NBI system.

by $D C$, which reduces the lateral displacement but induces oscillations in the wires, whose dynamic behaviour is however difficult to predict. A test stand has been built with the purpose of simulating the B field conditions of the W7-X stray B field and the TSP operation at the NBI in W7-X [4].

\section{Vacuum system of the IPP (AUG/W7-X) NBI system}

\subsection{System description and working principle}

The vacuum system consists per injector of a combination of fore pumps, two turbomolecular pumps and four large TSPs, developed at IPP. This combination of rotary pumps with metallic getter pumps has already been successfully used in other high vacuum systems [5] and in previous experiments at IPP. The metal sublimation technology provides a fast pumping speed and large capacity for the chemically active $\left(\mathrm{O}_{2}, \mathrm{CO} \ldots\right)$ and medium active gases $\left(\mathrm{H}_{2}, \mathrm{D}_{2}, \mathrm{~N}_{2}\right)$ [6]. The inert gases (mainly noble gases and $\mathrm{CH}_{4}$ ) are pumped solely by the turbo pumps at a lower pumping speed. 
In a TSP, Titanium is sublimed from ohmically heated wires (sublimators) and condenses on the surrounding surfaces, building fresh metallic layers. The gas molecules are captured due to complex physisorption, chemisorption and diffusion processes, whose performance depends on the molecule type, the temperature of the walls, gas composition, pressure and temperature, Ti layer structure, layer saturation level and impurities [7]. As more and more molecules are gettered, the Ti layer approaches saturation and the pumping speed decreases. A new layer has to be deposited.

\subsection{IPP TSP description}

The IPP TSPs have been in operation for the last 20 years in the AUG NBI with four TSPs per injector on the lateral sides of the NBI vacuum vessel. They operate at room temperature with a pumping speed for $\mathrm{H}_{2} / \mathrm{D}_{2}$ of up to $700 \mathrm{~m}^{3} / \mathrm{s}$ per pump $\left(2.8 * 10^{3} \mathrm{~m}^{3} / \mathrm{s}\right.$ in total), fulfilling the NBI system requirements [8]. A very large total surface is needed, which is achieved with a simple but effective design. The IPP TSP is $4.2 \mathrm{~m}$ high, $1.5 \mathrm{~m}$ wide and $0.25 \mathrm{~m}$ deep, divided into nine chambers by vertical walls hanging from a top flange (fig. 2 a)). These walls consist of water cooled aluminium panels which are corrugated to further increase the total surface and rough due to the sand casting production process, which enhances the adhesion of the first Ti layers. Inside each vertical chamber there are six $4 \mathrm{~m}$ long wires hanging from the top flange and connected in pairs. The wires are connected at the bottom through a partially ceramic insulated $\mathrm{Cu}$ braid loop to accommodate different wire elongation due to the thermal expansion (fig. 2 b) ). The design of the wire is inherited from a development at the French agency CEA in the 70's for similarly large TSPs [9]. It consists of a wire composite with a $\varnothing 2 \mathrm{~mm}$ tantalum core and two wire layers, the first with a $\varnothing 0.8 \mathrm{~mm}$ titanium wire and a $\varnothing 0.6 \mathrm{~mm}$ molybdenum wire wound around, with the Mo wire for stabilization, and the second layer with a $\varnothing 0.8 \mathrm{~mm}$ wound in the opposite direction Ti wire (fig. $2 \mathrm{c}$ )). The resulting $4 \mathrm{~m}$ wire weights aprox. $425 \mathrm{~g}$, has a large Ti content (around $200 \mathrm{~g}$ ), is relative flexible, can be cut to a desired length and is easier to handle than the more frequently used Ti-Mo rigid filaments in smaller-sized TSP.

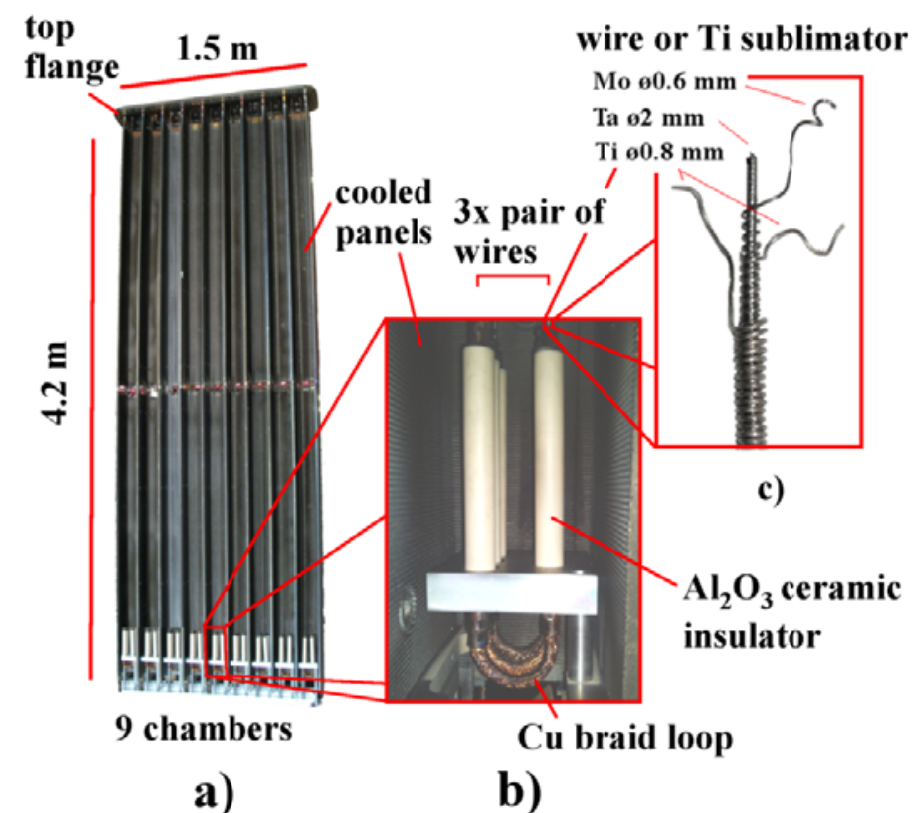

Figure 2: TSP overview with the bottom Cu braid loop system and the Ti sublimators. 


\subsection{IPP TSP standard operation}

The sublimation cycles of the NBI TSPs occur between AUG discharges (around 25 per day) in the relatively long pauses among them, avoiding so the interferences with the up to $30 \mathrm{mT}$ stray $B$ field of the Tokamak. In each TSP chamber only one of the three pairs of wires is heated at a time, until it cannot be used anymore and it must be switched to the next pair. In general, a whole sublimation sequence is performed through all chambers at the beginning of the experiment day. One or two chambers in all four pumps are coated with $\mathrm{Ti}$, changing the chambers sequentially one after another after every AUG pulse. The Ti sublimation must be pulsed to renew the Ti layers and recover the pumping speed that decreases as the Ti layers get saturated. The pair of wires is heated up with typically 142 A DC currents, corresponding to a heating power of around 30-32 kW, enough to raise the wire temperature up to $1900 \mathrm{~K}$ for 150 seconds. In the first and last $10 \mathrm{~s}$ the current is slowly ramped up and down correspondingly, to avoid abrupt current changes (see fig. 6 for the AC pulse).

For new wires, a conditioning process is performed by carefully increasing the heating current from cycle to cycle, up to $158 \mathrm{~A}$, until the wire resistance is reduced by around $10 \%$ [8]. A possible explanation for the reduction of the electrical resistance is that the wire temperature locally reaches the melting temperature of titanium. Consequently the Ti forms a more uniform external layer on the wire, which increases the wire's electric conductivity and provides a more uniform heat distribution and Ti sublimation.

\subsection{TSP Maintenance and repair}

Regular maintenance shutdowns are scheduled to coincide with normal AUG maintenance breaks with yearly frequency. One factor to be considered is the limited Ti reservoir in the TSP that must suffice for the required AUG-NBI operation. The presence of 3 pairs of wires per sublimation chamber extends the pumping operating time. Due to thermal expansion with the temperature increase, the wire's Ta core elongates a few $\mathrm{cm}$ during every pulse. After cooling down the wire does not completely return to the original length, an effect that accumulates and after some operational hours (3.5-11 h) the bottom Cu braid contacts the floor of the box during a pulse (max permanent lengthening around $0.11 \mathrm{~m}$ ) that may lead to mechanical failure or electric contact of the wires. The most likely explanation for this phenomenon is creep, during which the wire cross-section also narrows, eventually causing the wire to break off. If the copper braid contacts the floor or the wire lengthening is too different between the wires of a pair, a wire can bend and come in contact with the neighbouring wires, sticking to them. Should this occur and all three pairs of wires in a chamber are affected, then the whole chamber has been rendered useless, reducing the pumping performance of the whole TSP. The Ti reservoir in the wires is still large when the copper braid contacts the box floor, therefore the wires are shortened during maintenance and can be operated without restrictions till the next box floor contact.

\subsection{TSP in W7-X NBI}

The two front TSPs of the NBI system are installed in the closest region to the W7-X at $1 \mathrm{~m}$ range from its cryostat (fig. 1), where the permanent stray B field reaches around $30 \mathrm{mT}$. Unlike for other NBI components further away from the device, magnetic shielding with thick soft iron plates is not possible, since it would negatively influence the optimized B field configuration of W7-X. As a solution, heating by $A C$ instead of $D C$ has been proposed in order to reduce the wire deflection amplitude. However, the alternating excitation of the wires due to the interaction with the $B$ field 
produce wire accelerations estimated around $85 \mathrm{~m} / \mathrm{s}^{2}$ for sine waveform that could also have some negative effects on the operation of the TSP:

- Enhance critical fatigue or worsen the inherent wire lengthening, reducing their operation time.

- Cause large wire displacements if resonant frequencies are excited.

- Shake off Ti droplets from the wires during the sublimation due to the alternating force. This could change the structure of the deposited Ti layers, and thus influence the pumping properties.

The simulation of the involved phenomena by numerical models is too complex and it was decided therefore to proceed with the construction of a test stand.

\section{Test stand for the AC Operation of TSP in B field}

\subsection{Test stand setup}

The test stand for the TSP in B field is placed at a former NBI test stand at IPP Garching. The vacuum vessel is identical with those of the AUG-NBI and the test TSP is a modified spare pump. Of all 9 sublimation chambers, one is used with AC operation (fig. 3, position 3) and another with DC operation (fig. 3, position 7) in order to compare their behaviour. At the sides of the NBI box, windows have been installed behind the TSP at the bottom and at mid height that allow for direct observation of the wires (fig. 3, b), c) ).

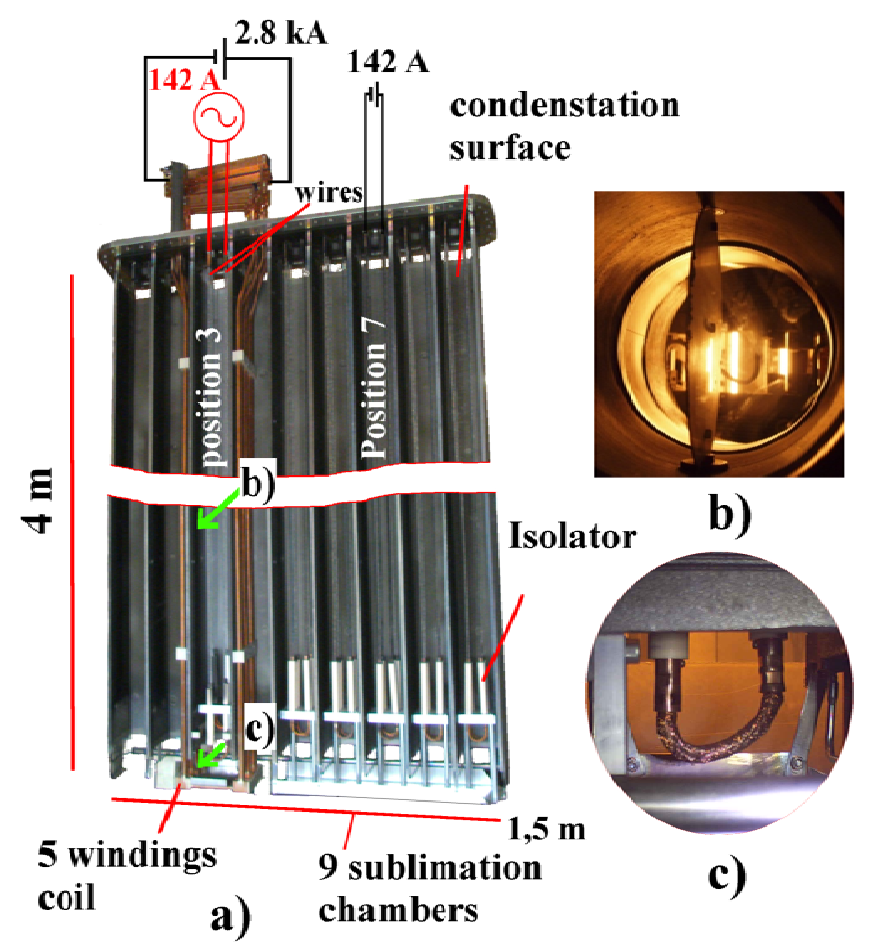

Figure 3: Modified TSP with the 5 windings coil. b) midplane view through the port with the manual shutter. c) bottom view for the Cu braid loop observation. 
A B field comparable to the one in W7-X is produced in the test stand by means of an elongated 5 windings coil made out of $\varnothing 20 \times 5 \mathrm{~mm}$ copper pipes which is mechanically fixed to the separating walls between chambers (2-3 and 3-4). The windings pass through electrical feedthroughs in the top flange to close the electrical circuit (fig. 3). The coil geometry was optimized using Quickfield(C to obtain a uniform B field up to $39 \mathrm{mT}$ inside the sublimation chamber for a coil current of $2800 \mathrm{~A} \mathrm{DC}$

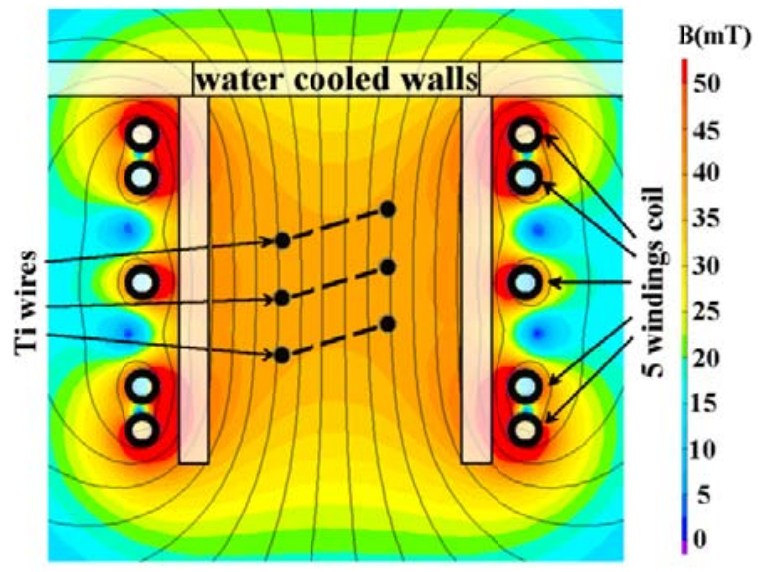

Figure 4: Horizontal cut-view of the simulated B field produced by the 5 windings coil with $2.8 \mathrm{kA}$ current in the sublimation chamber number 3 of the TSP.

(fig. 4). The B field produced by the wires itself is lower than $1 \%$ with respect to the one generated by the coil and its effect is considered here to be negligible.

A special AC power source has been installed that can deliver up to $50 \mathrm{~kW}$ for $260 \mathrm{~V}$ and allows a wide range of customization: frequency range from $60-1000 \mathrm{~Hz}$, different waveforms and programming of operation sequences.

\subsection{Diagnostics}

\section{Pressure observation}

With our current setup the evolution of the pressure inside the box is the only direct observable related to the pumping speed. The absolute pressure is measured by a Penning gauge. The background gas in the box is mainly residual air and outgassed molecules from walls and wires. For the first experimental stage no direct feed of $\mathrm{H}_{2}$ is considered, which will be performed later. Instead, a comparison is performed between the AC- and DC-operated sublimation chambers under the same operating parameters. The most relevant values to be observed are the ultimate pressure reached after many cycles and the evolution of the pressure level during and after every sublimation cycle.

\section{Ti deposition analysis}

The Ti layers deposited with DC and AC could present differences that may have an impact on the pumping effect if, for example, there is formation of Ti structures caused by the mentioned "shakeoff" effect of Ti droplets. The thickness of the deposited layers could deliver information on the layer history and the deposition rate. For this purpose, Al samples are installed as deposition probes at mid-height positions inside the sublimation chambers. During the maintenance openings of the box, 
they are replaced with new ones and are consequently analysed with metallographic tools for the surface and the Ti layer characteristics.

\section{Direct wire observation}

The wire vibration can be directly observed through a window, but only for short periods since the window is protected by a manual shutter to prevent it from getting covered with Ti. Through the bottom window it is possible to directly observe the thermal lengthening of the pair of wires during a pulse and also to quantify the permanent lengthening due to creep. The bottom glass has not direct sight of the wires and is therefore not covered by the sublimed Ti. A video camera is installed at the window to remotely observe the movement of the copper braid until it makes contact with the box.

\section{Wire dynamic behaviour - acceleration sensors}

A monitoring system for wire vibration was developed in order to detect the oscillation of the wires, which is transmitted to their fixations in the electrical feedthroughs of the top flange. It consists of tightly clamped supports around the insulating $\mathrm{Al}_{2} \mathrm{O}_{3}$ cylinders of the feedthrough each containing two acceleration sensors perpendicular to each other, with one oriented in the direction of the $B$ field as shown in figure 5. The stiffness of the construction allows the sensors to record the damped transverse oscillation of the wire's fixations as a qualitative measure of the wire oscillations.

The acceleration sensors contain piezoelectric crystals which are robust to electromagnetic noise and stable with respect to temperature changes. Their signals were processed in Labview ${ }^{\circledR}$ to obtain

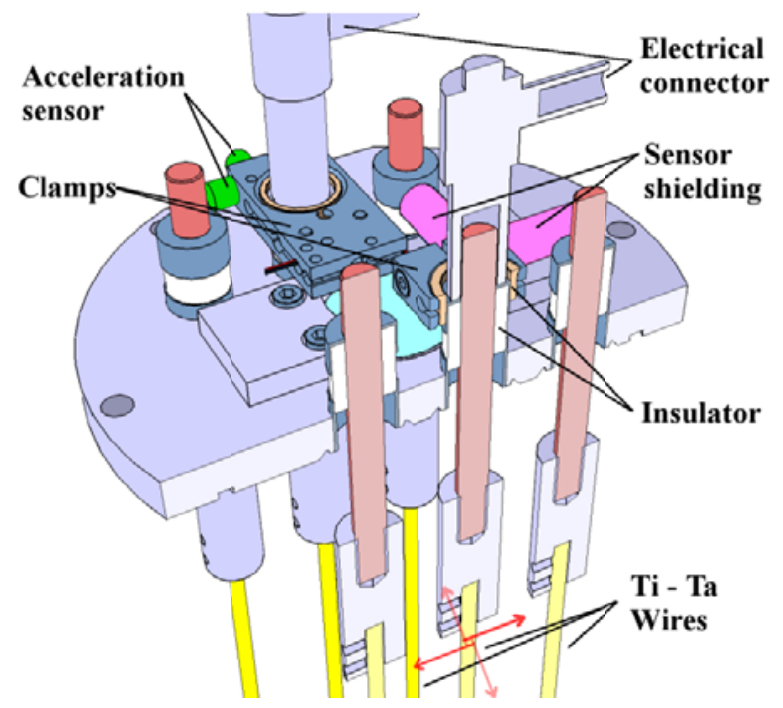

Figure 5: Cut view of the electric feedthrough with the acceleration sensors for the wire vibration detecting system.

frequency spectrum and signal intensity. The aim was to identify safe $A C$ parameters for the operation of the TSP with the W7-X stray B field conditions.

\subsection{Experimental program}

The first objective of the this experimental campaign was to demonstrate that $A C$ operation of the TSP was possible under $39 \mathrm{mT} B$ field conditions. After that, the effect of the oscillations on the wires 
in terms of wire reliability and pumping was tested. As a reference, the standard DC pulse parameters, sequences and procedures have been assumed. The AC pulse lasts $150 \mathrm{~s}$, where the current is ramped during $10 \mathrm{~s}$ at the beginning and end of the pulse and it is maintained constant for the rest of the time at $142 \mathrm{~A} \mathrm{RMS}$ averaged. In AC operation, the waveform and the frequency must be defined. Depending on the waveform, the wave peak to peak value is larger than the required RMS value: $\sqrt{2}$ larger for sine, $\sqrt{3}$ larger for triangle, while for square it is the same (crest factor definition). This could play a role in the dynamics of the wire since the instantaneous Lorentz force depends on the instantaneous current value. For the beginning, the sine waveform was chosen. Figure 6 shows a standard sublimation cycle with standard electric parameters and ramped frequency from 400 to $520 \mathrm{~Hz}$. The current is ramped in the first $10 \mathrm{~s}$ up to the nominal $142 \mathrm{~A}$. After $30 \mathrm{~s}$ the wires reach thermal equilibrium which can be observed in the stabilization of the voltage and electrical resistance. At that point, the frequency starts being ramped with typically $1 \mathrm{~Hz} / \mathrm{s}$ in order to avoid staying for a long time at potentially resonant frequencies. In the last $10 \mathrm{~s}$ the current is ramped down.

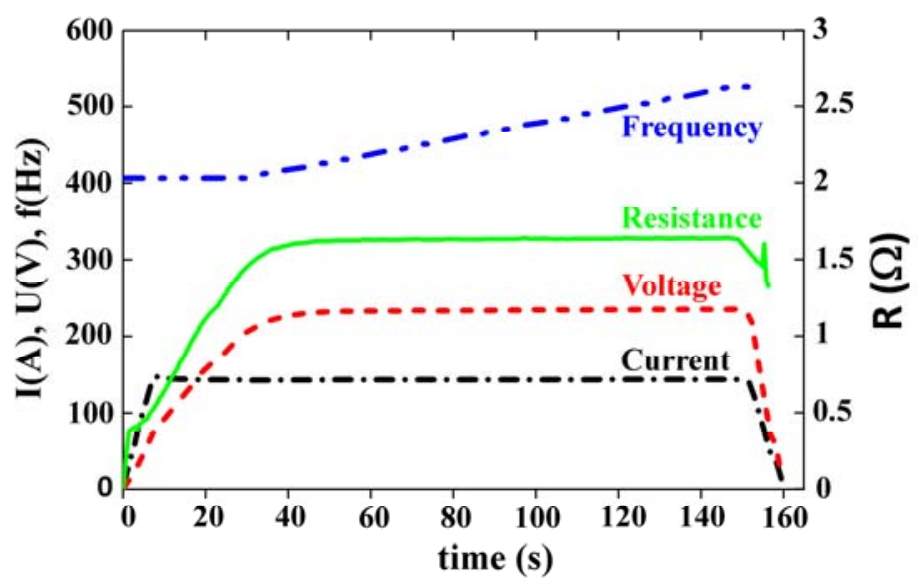

Figure 6: Standard cycle for $A C$ wire operation with ramped frequency variation from 400 to $520 \mathrm{~Hz}$. Left axis: current (I), voltage (U) and frequency $(f)$. Right axis: electrical resistance $(R)$.

Along this first campaign six experimental stages have been worked through:

1. First cycles were performed without B field. The heating current was increased subsequently in every pulse with sine waveform and a frequency ramp from 400 to $520 \mathrm{~Hz}$.

2. The B field strength was increased stepwise up to the maximum B field ( $39 \mathrm{mT}$ ) with standard AC heating current (142 A).

3. The conditioning sequence of new wires was tested with maximum B field.

4. The influence of the frequency was checked with frequency scans in the range 150$820 \mathrm{~Hz}$, in $120 \mathrm{~Hz}$ ramp intervals, upwards and downwards.

5. Sublimation cycles with square and triangle waveforms were performed, again with frequency scans.

6. In the end, tests under severe conditions were performed in order to test the dynamic reaction of the wires and the consequences of a sudden $A C$ current interruption, a B field 
change/interruption or successive pulse operation with constant frequency at high vibration amplitudes.

\section{Results}

\subsection{Electrical measurements}

Figure 7 shows a summary of the electrical parameters of the almost 800 sublimation cycles carried out with AC ohmically heated wires in the first experimental campaign. In total seven pairs of wires have been used. The number of the wire pair is indicated in the top of fig. 7. The first two pairs were previously used wires (indicated by \#). The remaining five pairs of wires, being new, had to be conditioned. This was performed successfully with the $39 \mathrm{mT} B$ field on. During the conditioning, the current, shown as blue circles, is increased stepwise up to $158 \mathrm{~A}$ until a reduction of $10 \%$ of the electric resistance, shown as green squares, is observed. Moreover two of the pairs of wires were utilized for a second time after their shortening (marked with *).

The nominal operation current was set to $142 \mathrm{~A}$, but a small variance of the measured current from this value is observed and caused by a dependency of the current control on frequency and waveform. This effect is not critical for the later operation and can easily be corrected. During wire operation at constant current, the electrical resistance increases fairly linearly with the number of sublimation cycles due to the wire lengthening and the consequent section narrowing. The values of the resistance in fig. 7 also show a large scatter between different pairs of wires, despite their identical construction. As a direct consequence, the required heating power for constant current operation (red columns) ranges from 23 to $35 \mathrm{~kW}$.

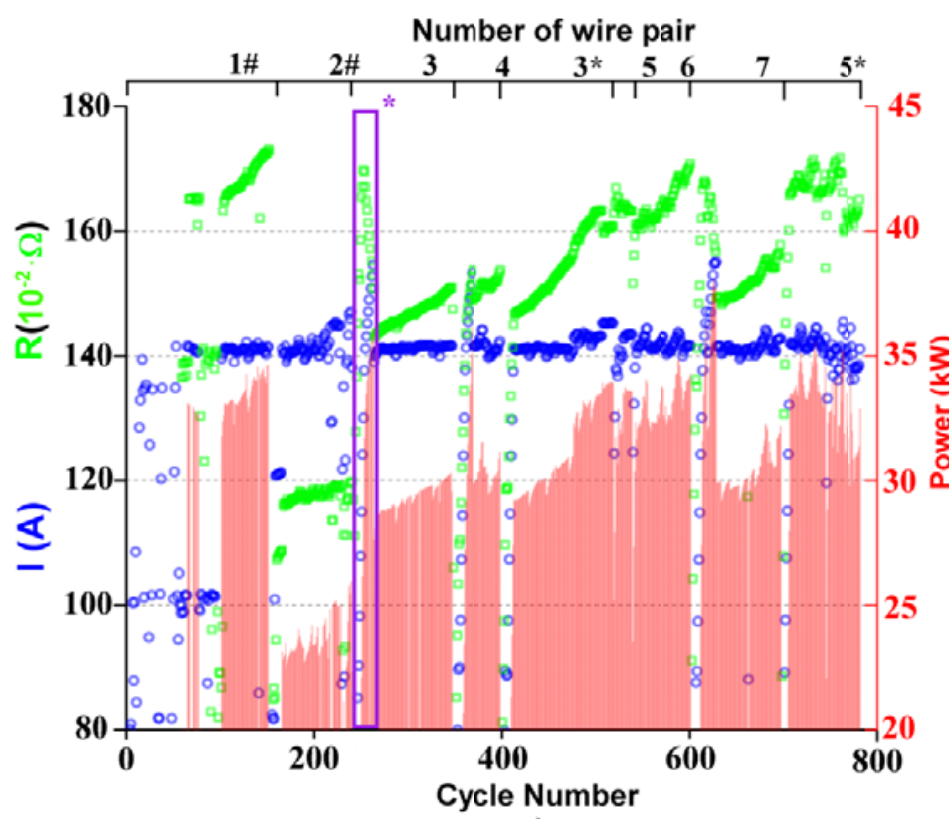

a)

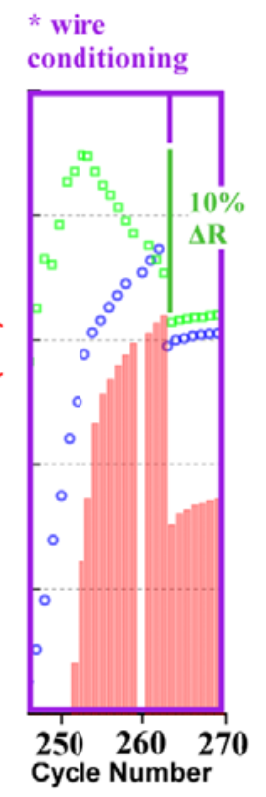

b)

Figure 7: a) Summary of the electrical measurements during the experimental campaign for 7 pairs of wires (\# already conditioned test wires - * shortened wires). b) Zoomed view of a wire conditioning sequence. 


\subsection{Pressure observations}

In figure 8, two conditioning sequences of used wires after venting of the vessel are shown for the $A C$ and $D C$ driven pairs of wires. The pressure at the beginning of the sequences was not at the same level and for the DC case the conditioning process was interrupted and continued on the next day, as can be seen from the break in the time axis. In the conditioning sequence the heating current is increased stepwise after each cycle, starting from around $40 \mathrm{~A}$. The wires and the surrounding walls outgas due to the heating and cause a pressure burst that is only pumped by the turbopumps. The shape of the pressure burst during the sublimation cycle changes as soon as Ti begins to sublime and consequently to pump, which happens for both AC and DC above $120 \mathrm{~A}$. From that moment the heating current is raised up to the standard value of $142 \mathrm{~A}$, while the pumping effect increase is observable after each heating cycle. After approximately the same number of AC and DC heating cycles, the pressure reached a similar pressure level of about $10^{-5} \mathrm{~Pa}$. During the experiments with both $A C$ and $D C$ operation, the ultimate measured pressure reached the mid $10^{-6}$ Pa region, which is the typical pressure level also in AUG-NBI after a long time of vacuum conditioning with many sublimation cycles with four TSPs.

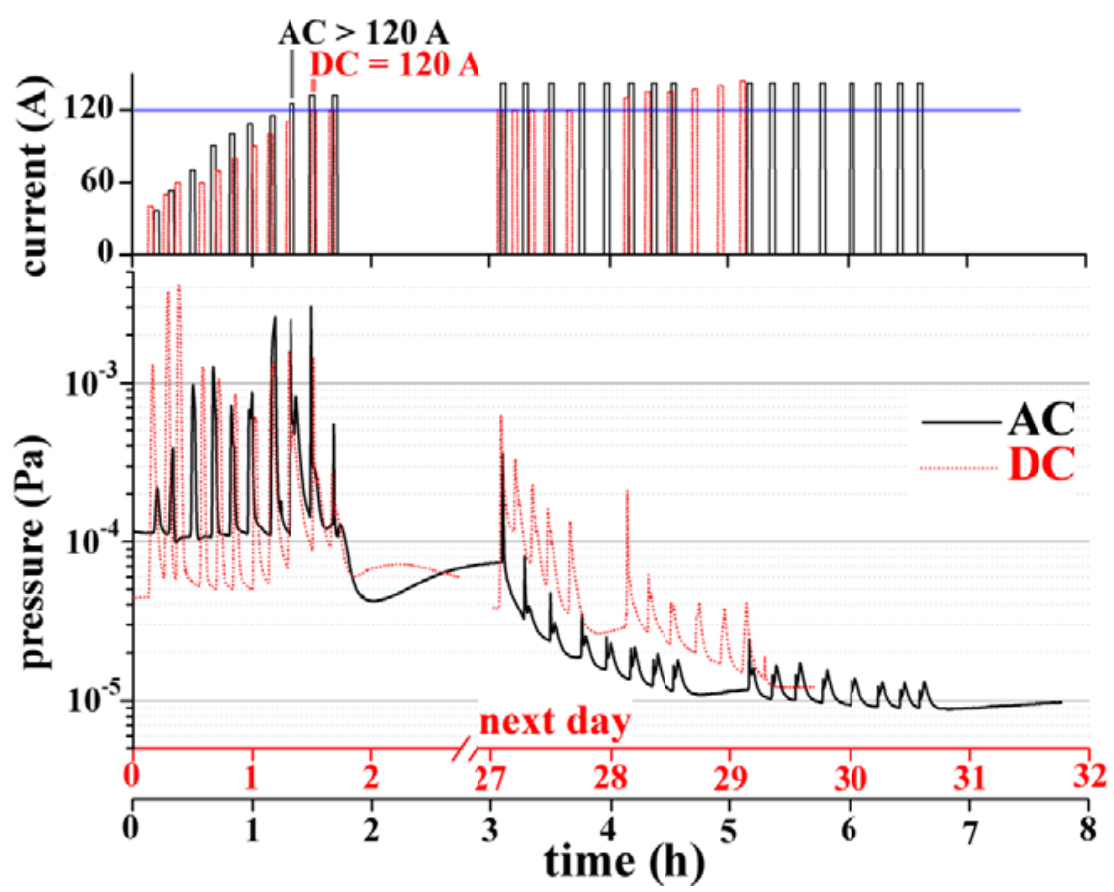

Figure 8: Pressure evolution for $A C$ and $D C$ operation on the first operation day after vessel venting and pumping down with the rotary pumps. For both $A C$ and $D C$ there is a pause after two operation hours, which in the case of DC operation was resumed on the following day.

\subsection{Ti layer characterization}

Samples from six complete sublimation sequences for both $A C$ and $D C$ have been studied. The surface was inspected with a scanning electron microscope (SEM) to visualize the structure of the deposited Ti layer. Over a broad range of scales it showed no remarkable difference in the surface roughness between the probes in the $D C$ operated chamber and the $A C$ operated chamber in the presence of a $39 \mathrm{mT}$ B field (fig. 9 bottom). In both AC and DC probes, equally rough surfaces were observed, which is required for proper pumping conditions [10]. No evidence of large droplet formation due to shake off was observed. 
The layer thickness, its structure and adhesion was analysed with a confocal laser microscope (CLSM), on a transverse cut of the samples (fig. 9 top). The deposited Ti layers revealed good adhesion to the Al substrate. Assuming constant Ti deposition rate, a rough estimate was calculated of 0.07 to $0.18 \mu \mathrm{m} /$ pulse for $D C$ and of 0.11 to $0.21 \mu \mathrm{m} /$ pulse for $A C$. According to the literature [7][10], a minimum deposition of $0.035 \mu \mathrm{m} /$ pulse is required for the maximum specific high pumping speeds and capacity. In both AC and DC, the films present voids and cavities (fig. 9 top) which can be attributed either to a poor layer deposition quality or damage that occurred after polishing of the sample.

The composition of the layer in different positions was studied with the energy-dispersive X-ray spectroscopy technique (EDS). The result showed mainly $\mathrm{Ti}$ concentrations from 80 to $90 \%$ in weight, as can be expected, with traces of oxygen (4-9\%) and nitrogen (1-2.5\%), which corresponds to the background air gases. There is however a rather large amount of compounds containing carbon (2.5 - 10\%), whose origin is to be clarified but most probably comes from impurities in the wires.
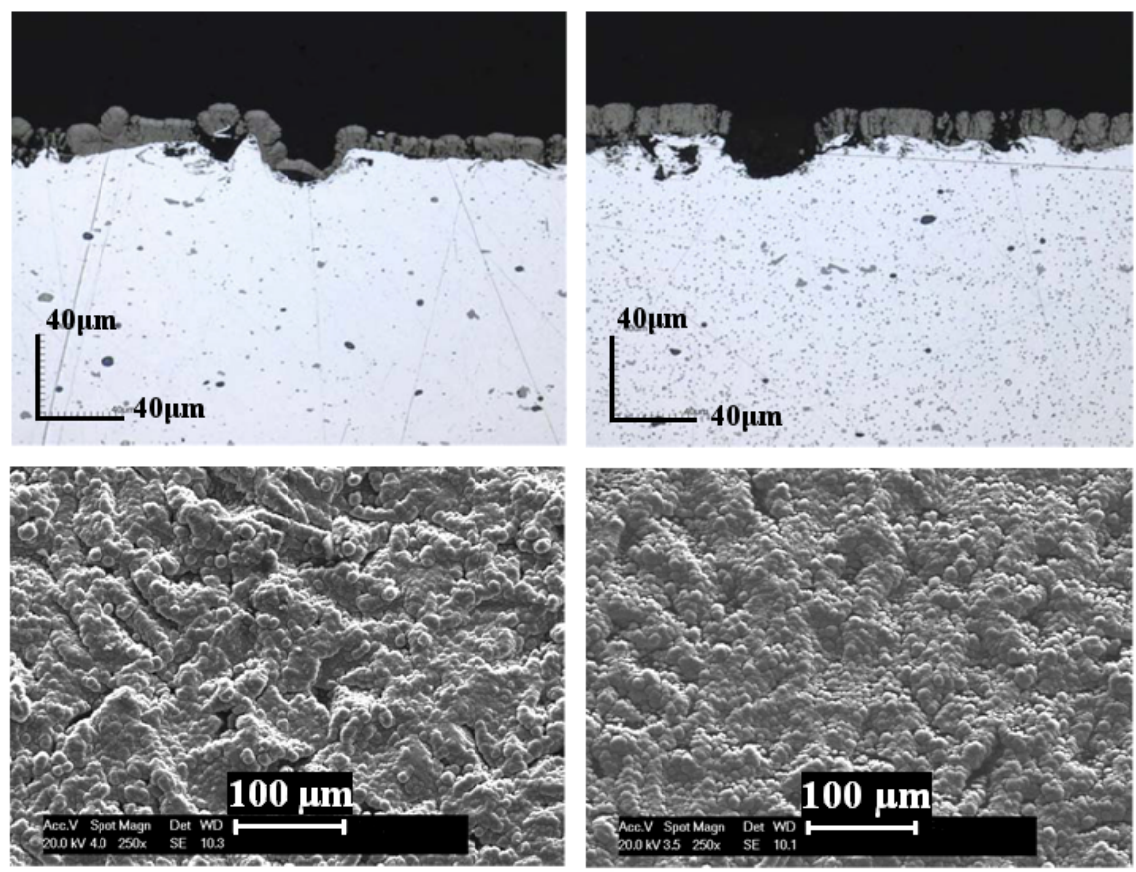

Figure 9: top: CLSM capture of the Ti layers - bottom: 250x magnified capture of SEM probe surface. Left side: AC operation, right side: DC operation.

\subsection{Results of the wire vibration monitoring system}

The wire vibration monitoring system with two perpendicular acceleration sensors per wire has been successfully employed for three pairs of wires. The measured accelerations are assumed to correspond to the acceleration of wire fixations inside the vessel and to some extent with wire oscillations. The interpretation of these results has been done in a qualitative and comparative way through the RMS value of the voltage signal from the sensors which corresponds $\left(1 \mathrm{~V}\right.$ to $\left.1 \mathrm{~m} / \mathrm{s}^{2}\right)$ to the sensor acceleration. Figure 10 shows the sensor response of three pairs of wires for sine and one of them also for square waveform with the frequency during multiple frequency scans in the range from 200 to $820 \mathrm{~Hz}$ for the nominal operation conditions. The signals of the sensors oriented 
perpendicularly to the B field are shown in the top panel of figure 10 while the bottom panel shows the parallel signals. Lines in the same colour correspond to the same pair of wires. From these measurements, an average value and its standard deviation, represented by the error bars, are calculated at each frequency. As can be observed, the sensor signals were repeatable with mostly low standard deviation for the same wire. The frequency response seems to be characteristic of each

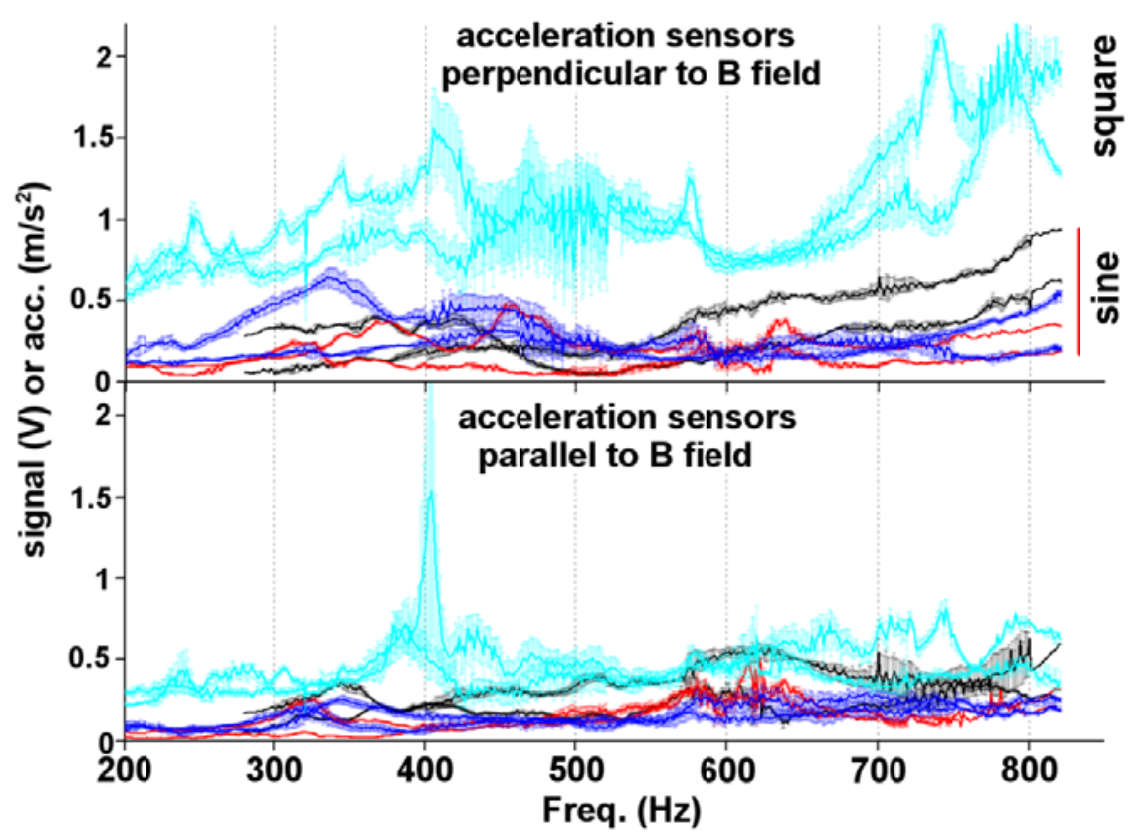

Figure 10: RMS value with the excitation frequency for 3 different pairs of wires (in black, red and dark blue) with sine waveform and $39 \mathrm{mT}$ B field. On top: acc. sensors oriented perpendicular to the $B$ field. on bottom: oriented parallel. The light blue curves correspond to the third pair of wires operated with square waveform.

wire. The largest standard deviation values are observed after many frequency scans. This is probably caused by the changes occurring in the wires such as the continuous wire lengthening and the mass change due to the Ti sublimation, which might slightly modify the wire dynamics.

Comparing the two sensor orientations, the perpendicular sensors showed larger signals, in agreement with the direction of the Lorentz force. A critical parameter is the waveform. For sine waveform, the maximum signal amplitude reached around $0.5 \mathrm{~V}$ in a central region from 300 to $700 \mathrm{~Hz}$. No clear pattern has been identified but local maxima appear at some frequencies for different wires in each direction. Under $300 \mathrm{~Hz}$ and over $700 \mathrm{~Hz}$, there seems to be a correlation of increasing sensor signals with increasing frequencies. The signal response of the square waveform, plotted in figure 10 (light blue) is up to three times larger than in the sine waveform perpendicular to the $B$ field. The explanation can be found in the spectral analysis.

By means of the "Short Time Fourier Transform" (STFT), time dependent spectrographs were calculated from the sensor signals. Figure 11 shows those spectra for the sine, square and triangular waveforms for standard pulse current and $39 \mathrm{mT} B$ field. In the first $30 \mathrm{~s}$ of the wire heating up, the frequency is kept constant at $400 \mathrm{~Hz}$ and then ramped up to $520 \mathrm{~Hz}$ with a $1 \mathrm{~Hz} / \mathrm{s}$ slope. In the presence of a $\mathrm{B}$ field, the acceleration sensor signals showed higher harmonics of the heating current frequency than without $B$ field. For the sine waveform (on top), the fundamental frequency is accompanied by many other higher harmonics. After $100 \mathrm{~s}$ the B field was interrupted in the sine 


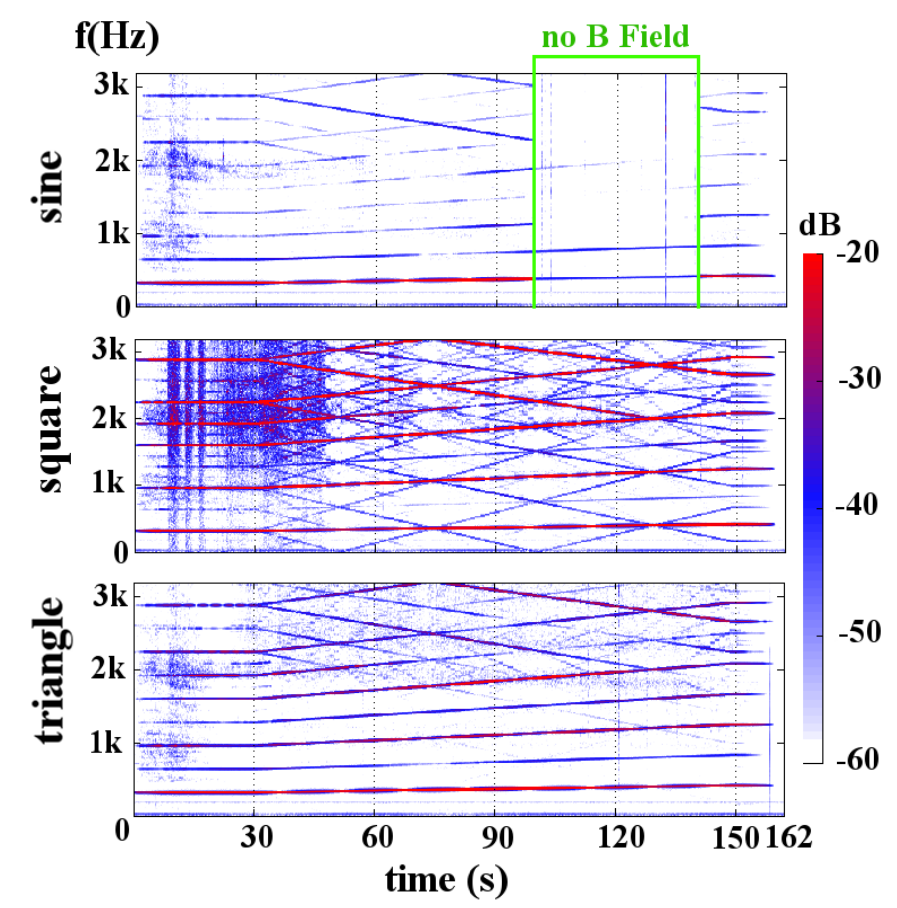

Figure 11: STFT spectrograms of three heating cycles for sine(top), square (middle) and triangle (bottom).

cycle, with a lower resulting signal and with only the fundamental frequency and the first harmonic. For square and triangle waveforms, the signal responses had many harmonics with amplitude sometimes even larger than the fundamental frequency. For these waves, however, new harmonic frequencies appeared with the ramped frequency change, both increasing and decreasing but with constant larger slope. The decreasing ramped frequencies are actually caused by the aliasing effect of the still higher harmonics (over $3.2 \mathrm{k} \mathrm{Hz}$ ) due to the selected sampling rate. Despite having theoretically lower instantaneous amplitude, the square waveform yielded higher signal amplitude values than sine waveform and triangle as can be observed in figure 10.

In order to test the resiliency of the wires, a pair of wires have been repeatedly operated in a frequency region where, at least in one direction it appeared to be a resonant frequency for square waveform (fig. 10 bottom at $405 \mathrm{~Hz}$ ). Even in this worst case no adverse behaviour of the wires has been encountered. The risk of failure is then believed to be lower with sine waveform. For that reason using the sine wave is appointed as the most appropriate choice.

The direct observation of the wires revealed in no case large directly observable deflections. However, a sub Hertz oscillation is observed after some events such as a sudden interruption of the heating current. This will be avoided during operation with a proper control system.

\subsection{Wire lengthening and lifetime}

The experience with the seven pairs of wires is that the operational time for AC- and DC-operated wires agrees well with the vast experience for the AUG-NBI with wires from the same delivery batch. Operating times of 3.5-4.5 h can be expected for new wires. After the shortening, further 6.5-7.5 hours of operation are possible until the Ti reservoir in the wires is almost depleted (fig. 12). The spread among the wires and specially for the different batches (as mentioned in [8]) is intrinsic to the wire design and an effect due to the manufacturing process, which cannot be easily controlled. 
In the end, this discrepancy obliges to schedule more frequent maintenance stops to shorten the wires and carefully estimate the wire length in order not to risk sticking of wires and losing pumping chambers.

The wire lengthening is faster at the beginning, followed a deceleration that becomes linear after a couple of operation hours (fig. 13). This linear behaviour remains after the wire shortening. The evolution of this behaviour fits with the theory of creep that has a primary phase with larger strain rate than in the secondary phase or steady state creep zone, where the elongation stabilizes. The tertiary creep phase has not been observed in the experiments, when the elongation accelerates due to local section narrowing that ends with breaking of the wire. This occurs however sometimes in the normal operation of the AUG-NBI.

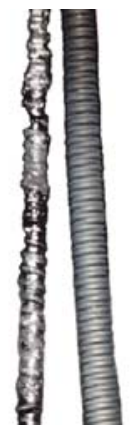

Figure 12: right: new Ti wire. left: almost depleted wire.

The different lengthening was also observed for each wire within a pair of wires [8]. The nonuniform lengthening of the wires in a pair of wires could easily lead to failure and for that reason this phenomenon should be studied in further detail.

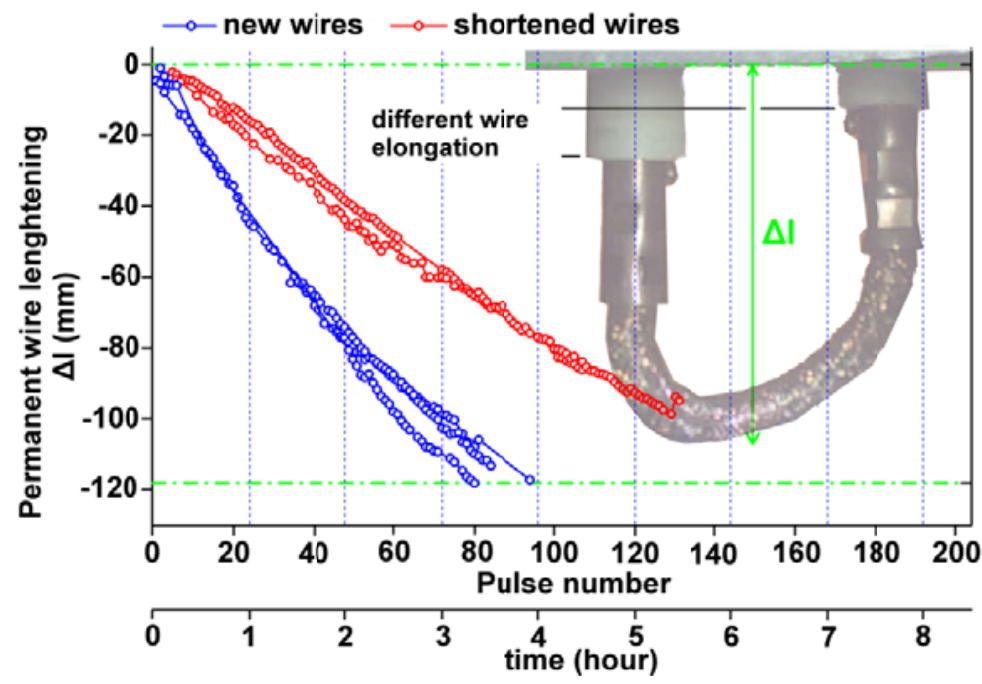

Figure 13: Residual wire lengthening attributed to creep as a function of the operation time for new and shortened wires. $\Delta l$ is the relative distance to the fix wall that can be observed from the bottom window.

\section{Conclusion}

The suitability of the AC operation for the TSP of the new NBI system of the W7-X, has been demonstrated in a dedicated test stand equipped with a modified IPP TSP. An experimental program has been carried out, performing around $800 \mathrm{AC}$ cycles with seven pairs of wires in a B field up to $39 \mathrm{mT}$ and no major event. By making use of $A C$ heating currents, the critical effect of the strong stray B field in the sublimators can be overcome for the long term operation of W7-X. The information gathered has allowed to compare AC- and DC-driven TSPs, showing no relevant difference with respect to the pumping effect, wire lengthening and wire reliability. The risks of the oscillating dynamic behaviour of the ohmically heated sublimators in presence of the B field have been measured with acceleration sensors and a safe and reliable operation of the pump is 
guaranteed. Based on the study of this dynamic behaviour a recommendation is given to operate with a sine waveform and a ramped frequency sequence during the pulse instead of a fix frequency, in order to avoid staying for a long time at possible resonant frequencies. No such resonant phenomena occurred with critical consequences during the experiments.

\section{Acknowledgments}

Special thanks for the support to Mrs. G. Matern from the department for metallography and to the ITED group at IPP.

This project has received funding from the EURATOM research and training programme 2014-2018.

\section{References}

[1] P. McNeely et al 2013 Fusion Eng. Des., Vol. 88, Issues 6-8, pp 1034-1037

[2] A. Staebler et al 1988 Fusion Technology, pp. 620-624

[3] M. Kick et al 2009 Fusion Eng. Des., Vol. 84, Issues 2-6, pp 1020-1025

[4] H.-S. Bosch et al 2013 Nucl. Fusion, Vol. 53, Issue 12, pp 12600

[5] R. Cyranski, J.H. Leck 1976 Vacuum, Vol. 26, Issue 9, pp 371-375

[6] A.K. Gupta, J.H. Leck 1975 Vacuum, Vol. 25, Issue 8, pp 362-372

[7] L. Elsworth 1965 Vacuum, Vol. 15, Issue 7, pp 337-345

[8] J.-H. Feist et al 1992 Proc. 17th SOFT Fusion Technology, Vol. 1, pp 262-266

[9] F. Prevot and Z. Sledziewski 1972 Vac. Sci. Technol., Vol. 9, pp 49

[10] G.I. Grigorov 1984 Vacuum, Vol. 34, Issue 5, pp 513-517 